\title{
Erratum to: Production of 10-hydroxystearic acid from oleic acid and olive oil hydrolyzate by an oleate hydratase from Lysinibacillus fusiformis
}

Bi-Na Kim • Young-Chul Joo • Yeong-Su Kim • Kyoung-Rok Kim $\cdot$ Deok-Kun Oh

Published online: 2 June 2012

(C) Springer-Verlag 2012

Erratum to: Appl Microbiol Biotechnol

DOI 10.1007/s00253-011-3805-2

Unfortunately, in the original publication of this article, Figures $1 \mathrm{a}$ and 2 are incorrect. The correct figure is given below:

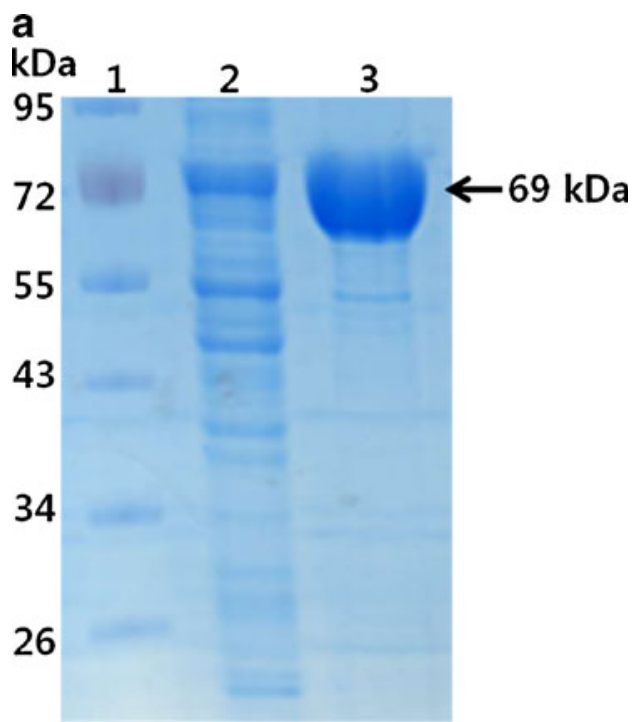

Fig. 1 SDS-PAGE analysis and molecular mass determination of oleate hydratase from $L$. fusiformis. a SDS-PAGE stained with Coomassie blue of the putative fatty acid hydratase from M. caseolyticus. Lane 1, molecular mass marker proteins $(95,72,55,43,34$, and $26 \mathrm{kDa})$; lane 2, crude extract; lane 3 , purified putative fatty acid hydratase

The online version of the original article can be found at http://dx.doi.org/ 10.1007/s00253-011-3805-2.

B.-N. Kim • Y.-C. Joo • Y.-S. Kim • K.-R. Kim • D.-K. Oh $(\bowtie)$

Department of Bioscience and Biotechnology, Konkuk University,

Seoul 143-701, Republic of Korea

e-mail: deokkun@konkuk.ac.kr 
Fig. 2 GC/MS spectrum of the silylated hydroxy fatty acid product obtained from oleic acid and linoleic acid by the putative fatty acid hydratase from $L$. fusiformis. The enzymatic silylated hydroxy fatty acid product was identified as 10-hydroxystearic acid

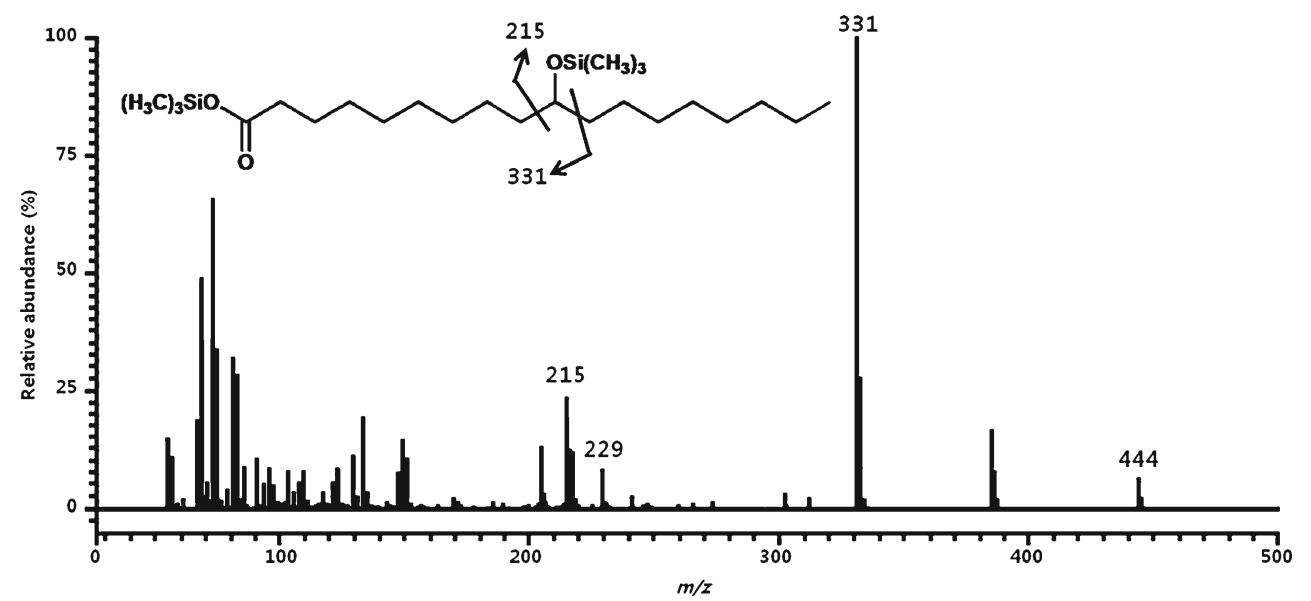

